\section{'Bhut Jolokia'-The World's Hottest Known Chile Pepper is a Putative Naturally Occurring Interspecific Hybrid}

\author{
Paul W. Bosland ${ }^{\mathbf{1}}$ and Jit B. Baral \\ Department of Plant and Environmental Sciences, New Mexico State \\ University, Las Cruces, NM 88003
}

Additional index words. aji, capsaicinoids, Capsicum chinense, Capsicum frutescens, pungency, RAPD, Scoville heat units

\begin{abstract}
In replicated trials at Las Cruces, N.M., the Scoville heat units (SHUs) of 'Bhut Jolokia', a chile pepper from Assam, India, reached one million SHUs. Morphologic characters revealed that 'Bhut Jolokia' is a Capsicum chinense Jacq. cultivar. Molecular analysis with randomly amplified polymorphic DNA markers confirmed the species identification and, interestingly, revealed that there may have been genetic introgression from Capsicum frutescens L. into 'Bhut Jolokia'.
\end{abstract}

Chile peppers (Capsicum L. spp.) are known for causing the sensation of heat or burning when consumed. The heat sensation is incited by the type and the amount of a group of capsaicinoids, the alkaloids found only in chile pepper pods (Zewdie and Bosland, 2001). The amount of capsaicinoids in a chile pepper pod is dependent on the genetic makeup of the plant and the environment where it is grown (Harvell and Bosland, 1997; Zewdie and Bosland, 2000). The capsaicinoids have evolved in chile peppers as a defense mechanism against mammalian predators (Tewksbury and Nabhan, 2001); nevertheless, this trait is an important fruit quality attribute and one of the most important reasons chile peppers are consumed.

Chile peppers were first introduced to Europe by Christopher Columbus. Shortly after the voyage of Columbus, Portuguese traders introduced chile peppers along their trade routes in Africa and Asia, including India (Andrews, 1999). By 1542, three varieties of chile peppers were recognized to be growing in India (Purseglove, 1968). Today, numerous landraces of chile pepper differing in shape, size, color, and heat level can be found in India as farmers selected chile peppers to fit their needs.

The northeastern region of India claims that the chile peppers grown in this region are the hottest in the world. Genetic resources of chile pepper landraces in northeastern India have not been well documented, but a few names mentioned include 'Naga Jolokia', 'Bhut Jolokia', and 'Bih Jolokia'. The Assamese word "jolokia" means the Capsicum

Received for publication 23 Sept. 2006. Accepted for publication 18 Nov. 2006.

A contribution of the New Mexico Agri. Expt. Sta., New Mexico State Univ., Las Cruces.

${ }^{1}$ To whom reprint requests should be addressed; e-mail pbosland@nmsu.edu. pepper. Mathur et al. (2000) reported the 'Naga Jolokia' to be a variety of C. frutescens L. and to have a very high heat level, i.e., 855,000 Scoville heat units (SHUs). The hottest chile pepper on record is the $C$. chinense Jacq. cultivar Red Savina with a heat level of 577,000 SHUs (Guinness Book of World Records, 2006).

This study was undertaken to 1) compare the heat levels of 'Red Savina', 'Bhut Jolokia', and habanero in a replicated field trial; 2) establish whether 'Bhut Jolokia' truly has a higher heat level than 'Red Savina'; and 3 ) determine the species designation of the 'Bhut Jolokia.'

\section{Materials and Methods}

A member who had collected it while visiting India sent the original seed of 'Bhut Jolokia' to the Chile Pepper Institute in 2001. Because of poor fruit and seed set, it took several years to have sufficient seed of the accession for a replicated field trial. 'Bhut Jolokia' was grown under insect-proof net cages to produce the bulk seed with the 2004 seed increase used in the replicated trials (Bosland, 1993). Seeds of 'Red Savina' (Renee's Garden Seeds, Felton, Calif.), a standard orange habanero (Seminis, Oxnard, Calif.), and 'Bhut Jolokia' were compared in 2005 at the Leyendecker Plant Science Research Center, $2.5 \mathrm{~km}$ south of Las Cruces, N.M.

Seeds were sown into a planting medium (Metro-Mix 360; Sun Gro Horticulture, Bellevue, Wash.) in 12-celled bedding plant containers (Hummert Intl., Earth City, Mo.) and placed in a greenhouse maintained at $27{ }^{\circ} \mathrm{C}$ day $/ 18{ }^{\circ} \mathrm{C}$ night $\pm 3{ }^{\circ} \mathrm{C}$ with a $12-\mathrm{h}$ photoperiod. For each accession, two seeds per cell were sown in each cell, and after germination, seedlings were thinned to a single plant per cell and fertilized with $\approx 1.5 \mathrm{~g}$ of a 3- to 4-month slow-release fertilizer
(Osmocote 14N-4.2P-6.2K). Seedlings were watered twice per day.

When seedlings had eight to 10 true leaves, they were transplanted to field plots in a randomized complete block design with five replications. Each replication had $\approx 36$ plants. Plants were transplanted $25 \mathrm{~cm}$ apart in a single row with $1 \mathrm{~m}$ between rows. The plants were grown using standard cultural practices for growing chile peppers in southern New Mexico (Bosland and Walker, 2004). Morphologic data for 'Bhut Jolokia' were collected from the plants grown in the field. The Capsicum descriptors developed by the International Plant Genetic Resources Institute (1995) were used for characterization.

Once the fruit had matured on the plants in the field, a single harvest of 25 random mature fruits from at least 10 plants in each replication was bulked. After harvest, the sample was dried and ground. The extraction of the capsaicinoids and the estimation of capsaicinoid amounts followed the highperformance liquid chromatography (HPLC) procedures for the short run method as described by Collins et al. (1995). The HPLC data were converted from parts per million to SHU by multiplying the parts per million by 16 . Samples of the ground fruits were also sent to two commercial laboratories, Southwest Bio-Laboratories (Las Cruces, N.M.) and Ag-Biotech (Gilroy, Calif.), for heat level analysis to validate our results.

For randomly amplified polymorphic DNA (RAPD) analysis, four accessions of $C$. annuum, seven accessions of $C$. chinense, and seven accessions of $C$. frutescens (Table 1) were planted in 4-L plastic pots and grown in the greenhouse for DNA extraction. These plant materials were acquired from National Plant Germplasm System, Regional Plant Introduction Station, Griffin, Ga., and the New Mexico Capsicum Accession collection.

When seedlings had eight to 10 true leaves, six plants were randomly selected

Table 1. List of Capsicum plant material used in the randomly amplified polymorphic DNA analysis.

\begin{tabular}{llc}
\hline Accession ID & \multicolumn{1}{c}{ Species } & Source \\
\hline NephtP3 & C. annuum & NMSU \\
NephP96 & C. annuum & NMSU \\
NephtP56 & C. annuum & NMSU \\
NMCA10164 & C. annuum & NMSU \\
Bhut Jolokia & C. chinense & NMSU \\
GRIF9302 & C. chinense & USDA, GRIN \\
GRIF9269 & C. chinense & USDA, GRIN \\
PI213918 & C. chinense & USDA, GRIN \\
PI260477 & C. chinense & USDA, GRIN \\
PI260522 & C. chinense & USDA, GRIN \\
PI438648 & C. chinense & USDA, GRIN \\
PI439442 & C. chinense & USDA, GRIN \\
NMCA40035 & C. frutescens & NMSU \\
PI593613 & C. frutescens & USDA, GRIN \\
PI195296 & C. frutescens & USDA, GRIN \\
PI355395 & C. frutescens & USDA, GRIN \\
PI439490 & C. frutescens & USDA, GRIN \\
PI441642 & C. frutescens & USDA, GRIN \\
PI441645 & C. frutescens & USDA, GRIN \\
\hline
\end{tabular}

NMSU = New Mexico State University, USDA; GRIN = USDA Germplasm Resources Information Network. 
from each accession for DNA isolation. DNA extraction, quantification, and RAPD procedures, including the polymerase chain reaction thermal cycling profile used in this study, are described by Baral and Bosland (2002). Operon primers used were OPP7, OPF6, OPAI1, OPAJ11, OPAD12, OPAJ14, OPA4, OPAD6, OPAK4, OPM4, OPAN11, OPAE19, and OPC2. Presence or absence of RAPD bands was scored manually. If a RAPD band was present in a particular accession, it was scored " 1 "; if the band was not present, it was scored " 0 ." A computer software, numeric taxonomy, and multivariate analysis system, NTSYSpc, analyzed the RAPD markers (Rohlf, 1998). The "Simqual" program of NTSYSpc uses an algorithm to calculate the Dice's similarity coefficient for all possible pairwise comparisons (Dice, 1945). The unweighted pair group with arithmetic averages method performed the cluster analysis (Sneath and Sokal, 1973).

\section{Results}

The growing season in 2005 was favorable for the production of fruits on all three chile pepper cultivars. The environment is known to affect the heat level of chile pepper cultivars (Harvell and Bosland, 1997). Having a replicated field trial with standard control cultivars allows for a better comparison of heat levels among cultivars. The HPLC analysis revealed that orange habanero had a mean heat level of 357,729 SHUs, which is in the range normally seen for this cultivar in Las Cruces, N.M. (Table 2). The results of the analysis for 'Bhut Jolokia' indicated that it possessed an extremely high heat level, 1,001,304 SHUs, whereas 'Red Savina' recorded a heat level of 248,556 SHUs. Independent tests confirmed this high level of heat for 'Bhut Jolokia' with 927,199 SHUs and 879,953 SHUs from Southwest Bio-Laboratories and Ag-Biotech, respectively.

The leaf surface of 'Bhut Jolokia' has the characteristic crinkle look like in other $C$. chinense varieties (Baral and Bosland, 2004). There were two pendent flowers per axil with creamy white corollas. The filaments were purple and the anthers were blue. The elongated fruits were 5 to $8 \mathrm{~cm}$ in length with an undulating surface (Fig. 1). The constriction between calyx and pedicel found in $C$. chinense was present on the 'Bhut Jolokia' fruit but was reduced (Fig. 1). Fruits matured from green to bright red.

The 13 DNA primers produced 136 reproducible and reliable polymorphic RAPD markers among the 19 accessions of

Table 2. Heat level in Scoville heat units for the three Capsicum chinense cultivars tested.

\begin{tabular}{lr}
\hline Bhut Jolokia & $1,001,304 \mathrm{a}$ \\
Red Savina & $248,556 \mathrm{~b}$ \\
Orange Habanero & $357,729 \mathrm{~b}$ \\
\hline Different letters indicate significant differences \\
$(P \leq 0.05)$ among cultivars according to \\
Duncan's test.
\end{tabular}

C. annuum L., C. frutescens, C. chinense, and 'Bhut Jolokia' (Fig. 2). The RAPD markers varied in size from 300 base pairs (bp) to $1800 \mathrm{bp}$. The RAPD marker, OPP $7_{800}$, was present only in 'Bhut Jolokia'. There were 24, 13, and 20 RAPD markers specific to C. frutescens, C. chinense, and C. annuum, respectively. When RAPD markers were scored in 'Bhut Jolokia', eight C. chinensespecific RAPD markers and three $C$. frutescens-specific RAPD markers were present. None of the $C$. annuum-specific RAPD markers were detected in 'Bhut Jolokia'.

The genetic similarities among and within the species were calculated by averaging the Dice's similarity values for each pairwise comparison. Cluster analysis results are graphically presented in the form of a dendrogram in Figure 3. There are three main clusters, each representing one of the Capsicum species, i.e., C. annuum, C. chinense, and $C$. frutescens. The $C$. annuum accessions included in this analysis grouped into a single cluster at a similarity index value of 0.86 . Similarly, the $C$. chinense accessions and the C. frutescens accessions grouped together to their species designation at similarity index values of 0.82 and 0.85 , respectively. The $C$. frutescens and $C$. chinense clusters merged at the similarity index value of 0.45 . The average genetic similarity between C. chinense and 'Bhut Jolokia' was 0.79 .

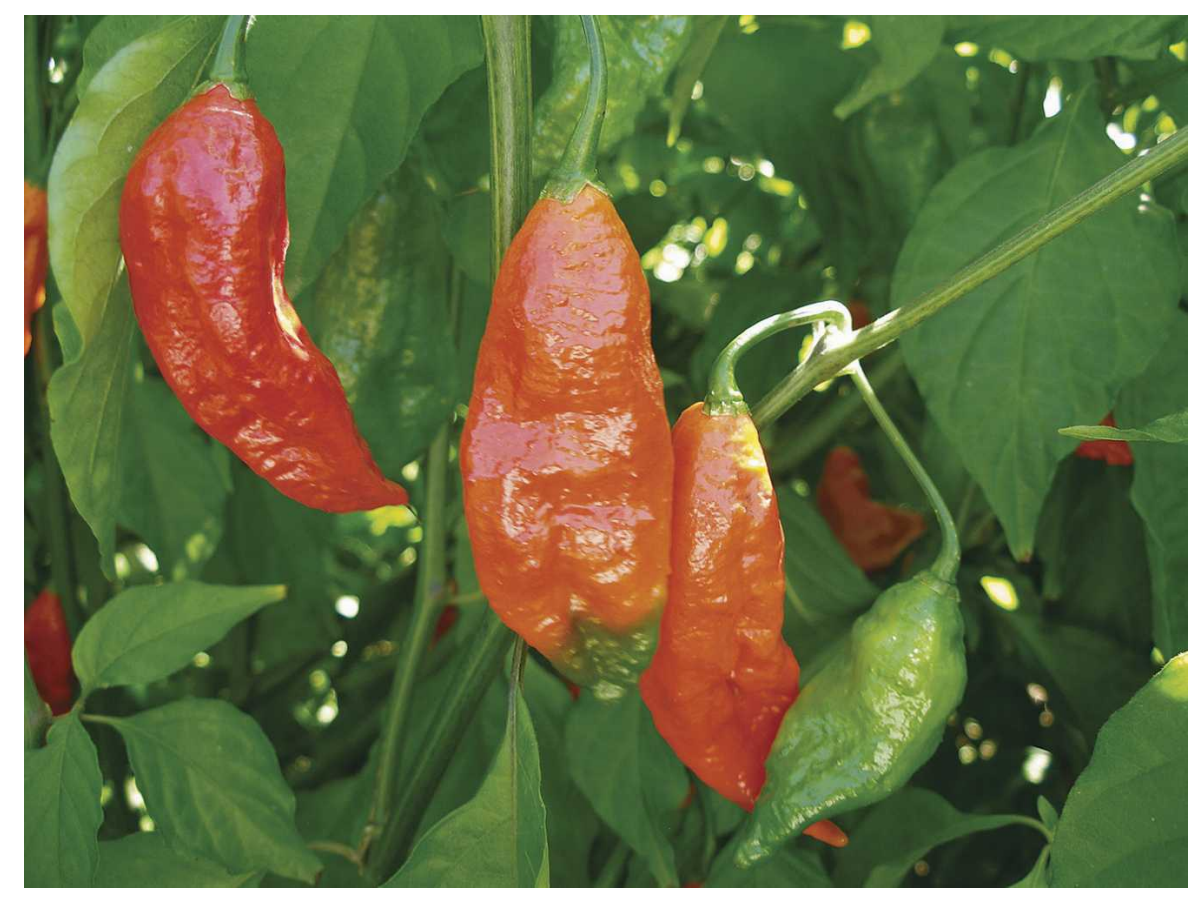

Fig. 1. Fruits of 'Bhut Jolokia' on plants grown in the field at the Leyendecker Plant Science Research Center.

\section{$\begin{array}{llllllllllllllllllllll}1 & 2 & 3 & 4 & 5 & 6 & 7 & 8 & 9 & 10 & 11 & 12 & 13 & 14 & 15 & 16 & 17 & 18 & 19 & 20 & 21 & 22\end{array}$}

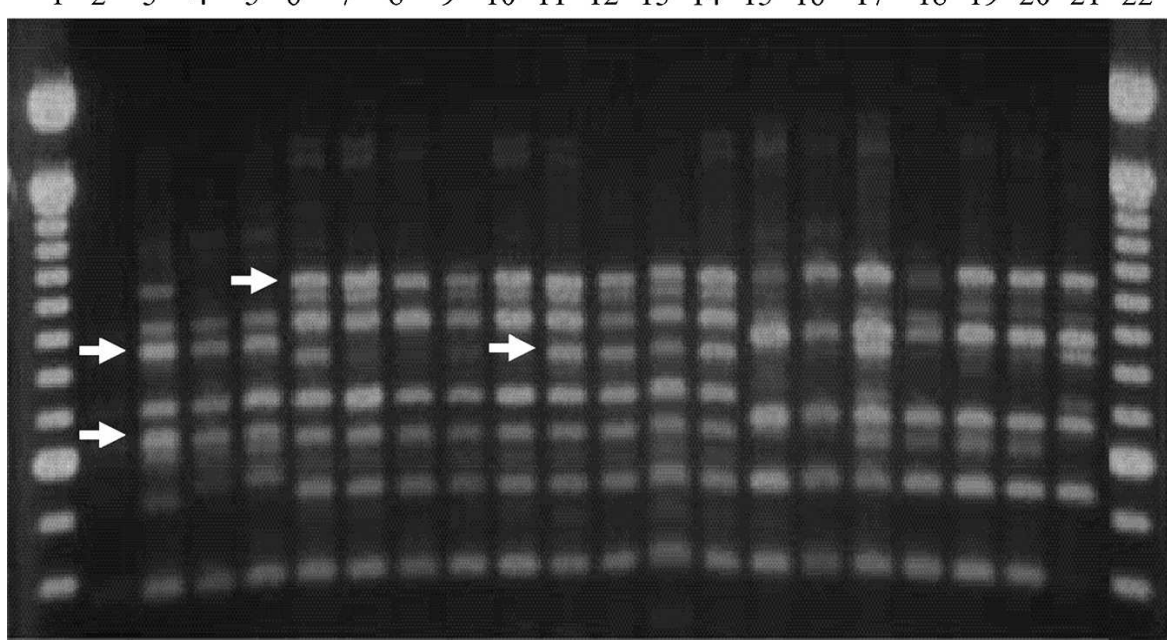

Fig. 2. Randomly amplified polymorphic DNA profiles generated by primer OPF6. Lane 2561 and 22, 100-base pair DNA ladder; lanes 2 to 5, Capsicum annuum; lanes 6 to 12, C. chinense; lanes 13 and 14, 'Bhut Jolokia'; lanes 15 to 21, C. frutescens. 


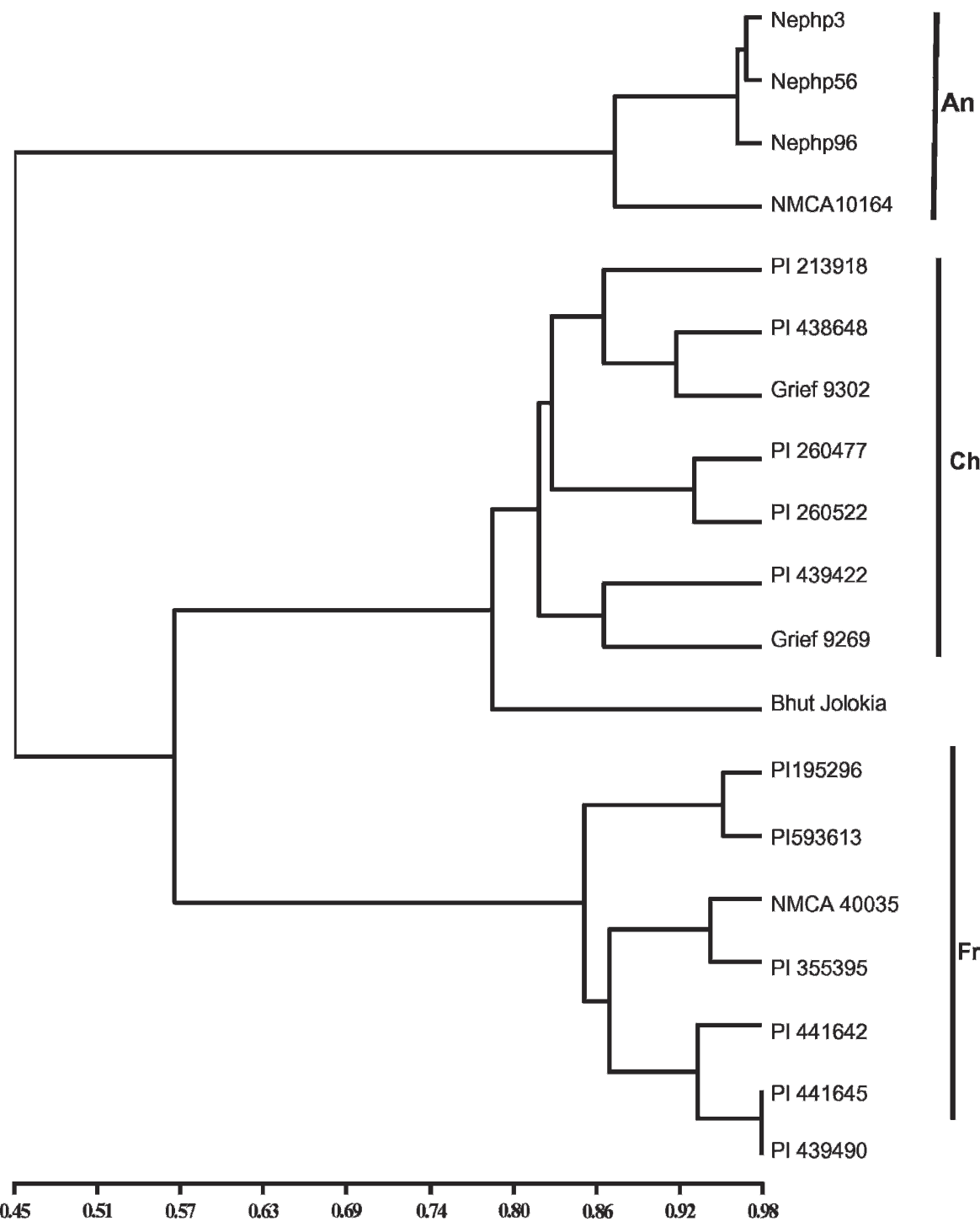

Fig. 3. Dendrogram based on unweighted pair group with arithmetic averages analysis of the Dice similarity coefficient among 19 Capsicum accessions using randomly amplified polymorphic DNA data. $\mathrm{An}=$ Capsicum annuum; $\mathrm{Ch}=C$. chinense; $\mathrm{Fr}=C$. frutescens.

\section{Discussion}

The fruit description of 'Naga Jolokia' given by the Defense Research Laboratory (Mathur et al., 2000) matched the plant material we received as 'Bhut Jolokia'. Based on the morphologic characters, i.e., presence of a calyx constriction, multiple flowers per node, whitish corolla, and pendent flower position, 'Bhut Jolokia' belongs to the $C$. chinense species, not $C$. frutescens. This is not surprising because in the past, the hottest chile peppers have belonged to $C$. chinense.

A widely used heat measurement of chile peppers is the SHU (Scoville, 1912). This measurement is the highest dilution of a chile pepper extract at which heat can be detected by a taste panel. Alternative instrumental methods have been developed since Scoville's test. HPLC is the most accurate and efficient method (Wall and Bosland, 1998). The extreme high heat level of 'Bhut Jolokia' was consistent over the past 3 years in nonreplicated tests. In addition, the heat mea-
C. frutescens. The presence of RAPD markers in 'Bhut Jolokia' that are specific to $C$. chinense and $C$. frutescens suggests an interspecific origin for 'Bhut Jolokia'.

Seed of 'Bhut Jolokia' is available through the Chile Pepper Institute, P.O. Box 30003 , MSC 3Q, NMSU, Las Cruces, NM 88003.

\section{Literature Cited}

Anderson, E. 1949. Introgressive hybridization. John Wiley and Sons, N.Y.

Andrews, J. 1999. The pepper trail. Univ. North Texas Press., Denton, Texas.

Baral, J. and P.W. Bosland. 2002. Genetic diversity of a Capsicum germplasm collection from Nepal as determined by randomly amplified polymorphic DNA markers. J. Amer. Soc. Hort. Sci. 127:316-324.

Baral, J.B. and P.W. Bosland. 2004. Unraveling the species dilemma Capsicum frutescens and C. chinense (Solanaceae): A multiple evidence approach using morphological, molecular analysis, and sexual compatibility. J. Amer. Soc. Hort. 129:826-832.

Bosland, P.W. 1993. An effective plant field-cage to increase the production of genetically pure chile (Capsicum spp.) seed. HortScience 28:1053.

Bosland, P.W. and S. Walker. 2004. Growing chiles in New Mexico. Cooperative Ext. Guide H-230.

Collins, M.D., L.M. Wasmund, and P.W. Bosland. 1995. Improved method for quantifying capsaicinoids in Capsicum using high-performance liquid chromatography. HortScience 30:137-139.

Dice, L.R. 1945. Measures of the amount of ecological association between species. Ecology 26:297-302.

Guinness Book of World Records. 2006. Hottest Spice. www.guinnessworldrecords.com. Accessed 13 Sept. 2006.

Harvell, K.P. and P.W. Bosland. 1997. The environment produces a significant effect on pungency of chiles (Capsicum annuum L.). HortScience 32:1992.

International Plant Genetic Resources Institute (IPGRI). 1995. Descriptors for Capsicum. Intl Plant Genetic Resources Inst., Rome.

Mathur, R., R.S. Dangi, S.C. Das, and R.C. Malhotra. 2000. The hottest chilli variety in India. Curr. Sci. India 79:287-288.

Purseglove, J.W. 1968. Tropical crops: Dicotyledons. Longman Group Ltd, London.

Rohlf, F.J. 1998. Numerical taxonomy and multivariate analysis system (NTSYSpc): Ver.2.0 Exeter Publication, Setauket, N.Y.

Scoville, W.L. 1912. Note on Capsicum. J. Amer. Pharm. Assoc. 1:453.

Sneath, P.H.A. and R.R. Sokal. 1973. Numerical taxonomy. W.H. Freeman and Co., San Francisco, Calif.

Tewksbury, J.J. and G.P. Nabhan. 2001. Directed deterrence by capsaicin in chilies. Nature 412:403-404

Wall, M.M. and P.W. Bosland. 1998. Analytical methods for color and pungency of chiles (Capsicum), p. 347-373. In: G. Charalambous (ed.). Instrumental methods in food and beverage analysis. Elsevier Science Publishers, Amsterdam

Zewdie, Y. and P.W. Bosland. 2000. Evaluation of genotype, environment, and genotype-by environment interaction for capsaicinoids in Capsicum annuum L. Euphytica 111:185-190.

Zewdie, Y. and P.W. Bosland. 2001. Capsaicinoid profiles are not good chemotaxonomic indicators for Capsicum species. Biochem. Syst. Ecol. 29:161-169. 